\title{
A rare case of a pancreatic mass due to accessory spleen; when EUS-FNA is not enough
}

A 56-year-old man was referred with asymptomatic elevation of pancreatic hydrolase levels. Magnetic resonance imaging (MRI) delineated a pancreatic lesion with a low T1 and high T2 signal ( Fig. 1). Endoscopic ultrasound (EUS) found an oval, well-defined, isoechogenic, homogeneous mass in the pancreatic parenchyma, without any vascular invasion and no locoregional lymph nodes ( $\bullet$ Fig. 2).

Fine-needle aspiration (FNA) showed small epithelioid cells. Immunostaining was positive for antichromogranin, antisynaptophysin, and anti-KI-67 (5\%), and a few cells were positive for anti-CD56. This was consistent with a neuroendocrine tumor (NET).

Octreotide positron emission tomography combined with computed tomography (PET-CT) showed a focal uptake into the pancreas without any other nonphysiological uptake ( Fig. 3).

CA19-9 and chromogranin levels were normal.

Caudal pancreatectomy with spleen preservation was performed. Histological examination found no proof of NET but did reveal an intrapancreatic accessory spleen (IPAS) ( $\bullet$ Fig. 4). The postoperative period and follow-up were satisfactory.

Accessory spleens may be found in $15 \%$ of the population but are rarely located in the pancreatic tail (17\%) [1]. Most IPASs have a homogeneous contrast-enhanced appearance on CT and MRI, sharing features with hypervascular lesions (such as NETs) [1].

Octreotide scans have a high sensitivity for detection of gastrointestinal NET (70\%$95 \%$ ). The somatostatin receptors on the surface of splenic lymphocytes may lead to false diagnosis of NET [2]. Nuclear scintigraphic investigations such as those with 99mTc sulfur colloid can help in identifying IPAS [3].

EUS findings include regular margins and homogeneous echogenicity, ranging from hypoechoic to hyperechoic [4].

FNA reveals small lymphocytes and a mixed inflammatory infiltrate with the appearance of white pulp. Sampling of islet cell clusters from the adjacent pancreatic parenchyma can lead to misdiagnosis.

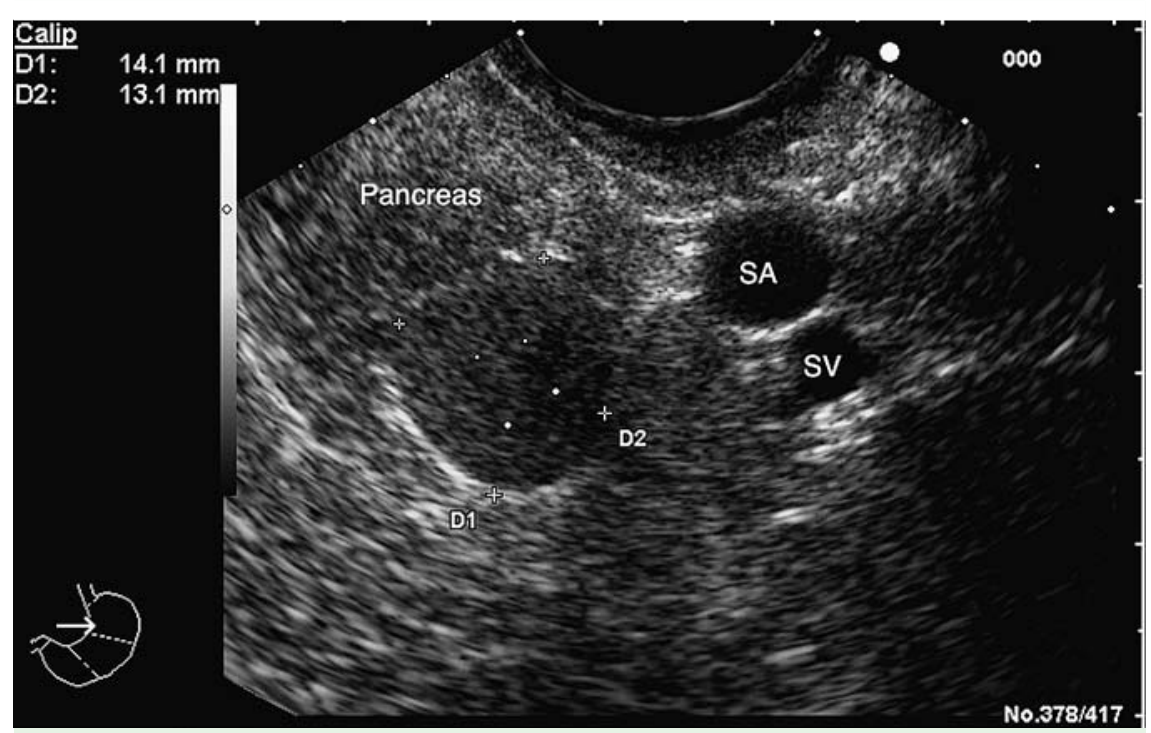

Fig. 1 Endoscopic ultrasound shows an oval, well-defined, isoechogenic, homogeneous, 14-mm mass located in the pancreatic tail. There is no cystic component or calcification (SA, splenic artery; SV, splenic vein).

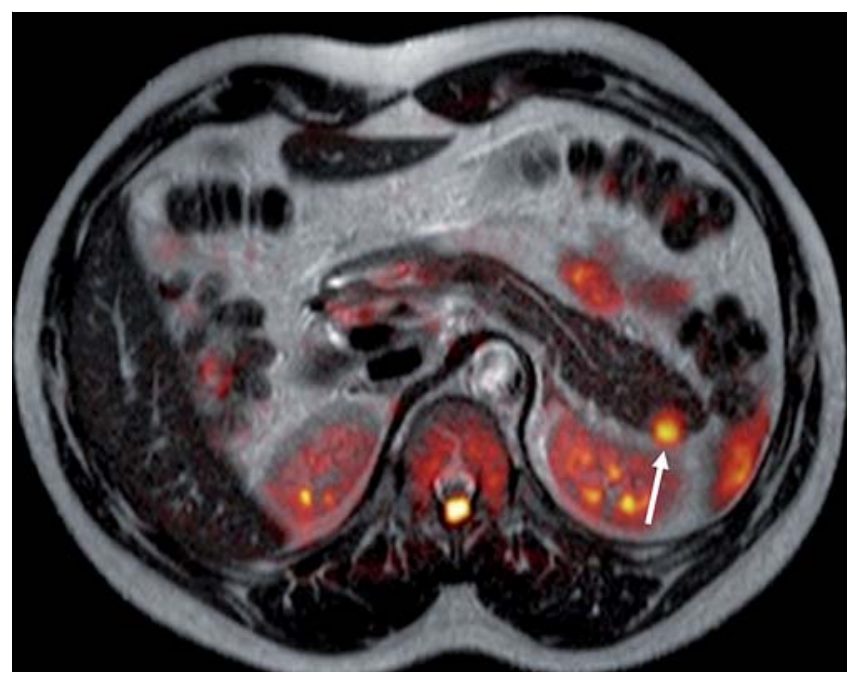

Fig. 2 Axial coregistration of turbo spin echo (TSE) T2-weighted and diffusion-weighted sections shows a well-defined and very bright nodule in the tail of the pancreas (arrow).

CD8 immunostaining of splenic sinus endothelial cells can help in confirming the diagnosis, as done retrospectively on FNA material in our patient [5].

Ultrasound endoscopists should be aware of this entity (IPAS) in order to avoid unnecessary surgery, even when FNA shows cells with NET characteristics.

Endoscopy_UCTN_Code_CCL_1AF_2AG_3AB

Competing interests: None
E. Toussaint ${ }^{1}$, P. Flamen ${ }^{2}$, P. Demetter ${ }^{3}$, C. Matos $^{4}$, M. Van Gossum ${ }^{5}$, M. Delhaye ${ }^{6}$, J. Closset ${ }^{7}$, P. Loi ${ }^{7}$, J. Deviere ${ }^{6}$, M. Arvanitakis $^{6}$

Medicine Department, Jules Bordet Institute, Brussels, Belgium

2 Nuclear Studies Department, Jules Bordet Institute, Brussels, Belgium 3 Pathology Department, Erasme University Hospital, Brussels, Belgium

4 Radiology Department, Erasme University Hospital, Brussels, Belgium ${ }^{5}$ Clinic of Hepato-gastroenterology, $\mathrm{CHU}$ Saint Pierre, Brussels, Belgium 

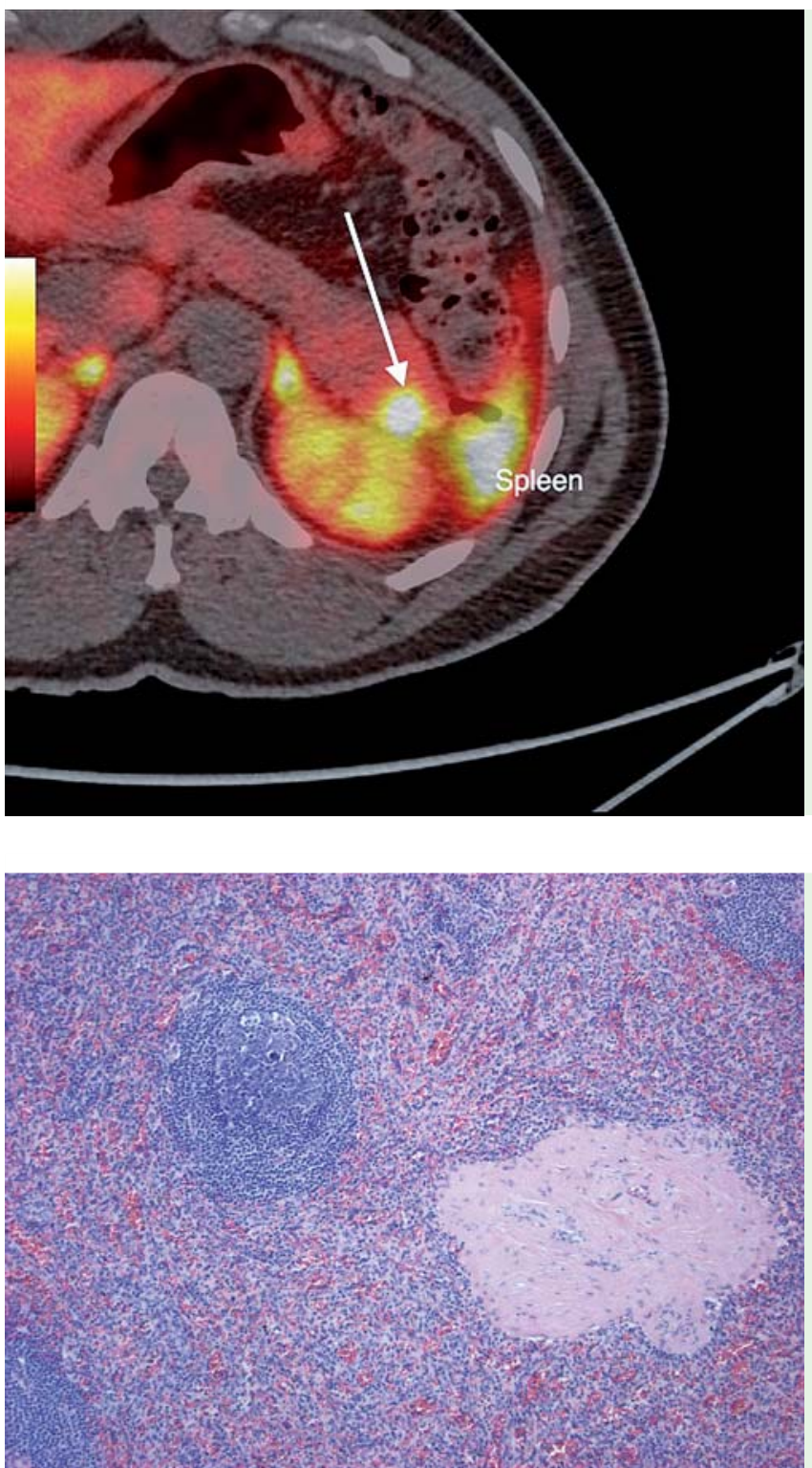

Fig. 3 Octreotide positron emission tomography combined with computed tomography (PET-CT) showing focal uptake in the pancreatic tail (arrow) suggestive of neuroendocrine tumor.

6 Medical Department of Gastroenterology, Erasme University Hospital, Brussels, Belgium

7 Surgical Department, Erasme University Hospital, Brussels, Belgium

\section{References}

1 Arkadopoulos N, Athanasopoulos P, Stafyla V et al. Intrapancreatic accessory spleen issues: diagnostic and therapeutic challenges. JOP 2009; 10: 400-405

2 Lebtahi R, Cadiot G, Marmuse JP et al. Falsepositive somatostatin receptor scintigraphy due to an accessory spleen. J Nucl Med 1997; 38: 1979-1981

3 Brasca LE, Zanello A, De Gaspari A et al. Intrapancreatic accessory spleen mimicking a neuroendocrine tumor: magnetic resonance findings and possible diagnostic role of different nuclear medicine tests. Eur Radiol 2004; 14: $1322-1323$

4 Barawi M, Bekal P, Gress $F$ et al. Accessory spleen: a potential cause of misdiagnosis at EUS. Gastrointest Endosc 2000; 52: 769 772

5 Lin J, Jing $X$. Fine-needle aspiration of intrapancreatic accessory spleen, mimic of pancreatic neoplasms. Arch Pathol Lab Med 2010; 134: $1474-1478$

Fig. 4 Histological image (hematoxylin and eosin [H\&E] staining) of the intrapancreatic splenic tissue.

\section{Bibliography}

DOI $10.1055 / \mathrm{s}-0030-1256421$

Endoscopy 2011; 43: E221 -E222

(c) Georg Thieme Verlag KG Stuttgart · New York . ISSN 0013-726X

\section{Corresponding author}

\section{E. Toussaint, MD}

Gastroenterology

Clinic of Medical Oncology

Institut Jules Bordet

1 rue Héger-Bordet

1000 Brussels

Belgium

Fax: +32-2-5380858

emmanuel.toussaint@bordet.be 